\title{
Cuidados de enfermagem ao paciente com mucopolissacaridose VI: uma revisão
}

\section{integrativa da literatura}

\author{
Nursing care for patients with mucopolysaccharidosis VI: an integrative literature review \\ Atención de enfermería a pacientes con mucopolisacaridosis VI: revisión integradora de la
}

literatura

Recebido: 17/11/2021 | Revisado: 19/11/2021 | Aceito: 17/12/2021 | Publicado: 01/01/2022

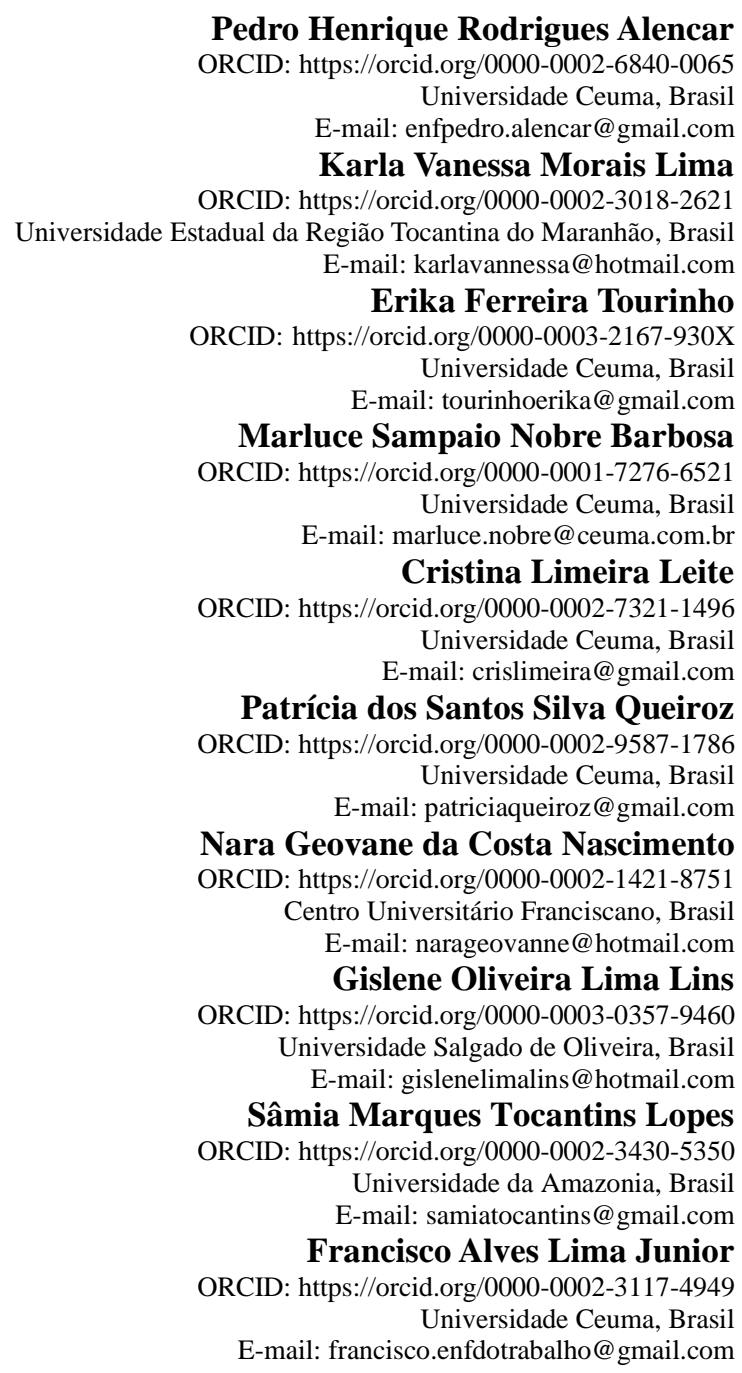

\begin{abstract}
Resumo
Objetivo: Evidenciar as necessidades dos pacientes portadores da Síndrome de Maroteaux-Lamy ou mucopolissacaridose VI, e diante disso propor os possíveis diagnósticos de enfermagem. Metodologia: A pesquisa é de natureza básica, cunho descritivo, de revisão de literatura. Nesse sentido, os dados foram coletados a partir de artigos da Scielo, BVS e LILACS, entre os anos de 2002 a 2015, onde durante a coleta de dados foram encontrados 1.242 artigos, e durante o processo de seleção, após todos os filtros, ficaram 7 artigos ao todo. Diante disso, a partir desses 7 artigos foram coletas as informações necessárias para elaboração de um quadro de possíveis diagnósticos de enfermagem. Resultado: Os pacientes portadores da MPS VI (mucopolissacaridose VI) tem uma maior prevalência para os comprometimentos osteoarticulares, conforme os dados coletados e analisados. Portanto, os possíveis diagnósticos de enfermagem podem ser mobilidade física prejudica, deambulação prejudicada, capacidade de transferência prejudicada, levantar-se prejudicada, sentar-se prejudicada. Conclusão: A partir das manifestações clínicas achadas na revisão literária, os possíveis diagnósticos de enfermagem têm intuito de maximizar a eficácia da
\end{abstract}


assistência de enfermagem, já que o diagnóstico de enfermagem é uma etapa crucial para elaborar um plano de ação, para o processo de cuidado do paciente. Logo, o enfermeiro tem esse papel importantíssimo com SAE (Sistematização da Assistência de Enfermagem), já que trata de uma das suas diversas atribuições.

Palavras-chave: Mucopolissacaridose VI; Síndrome de Maroteaux-Lamy; MPS-VI; Enfermeiro.

\begin{abstract}
Objective: To highlight the needs of patients with Maroteaux-Lamy Syndrome or mucopolysaccharidosis VI, and in the light of this to propose possible nursing diagnoses. Methodology: The research is of a basic nature, descriptive, literature review. In this sense, data were collected from articles in Scielo, BVS and LILACS, between the years 2002 to 2015, where during data collection 1,242 articles were found, and during the selection process, after all filters, they remained 7 articles in all. Therefore, from these 7 articles, the necessary information was collected to elaborate a framework of possible nursing diagnoses. Result: Patients with MPS VI (mucopolysaccharidosis VI) have a higher prevalence of osteoarticular involvement, according to the data collected and analyzed. Therefore, possible nursing diagnoses can be impaired physical mobility, impaired walking, impaired transferability, impaired getting up, impaired sitting. Conclusion: Based on the clinical manifestations found in the literature review, the possible nursing diagnoses aim to maximize the effectiveness of nursing care, since the nursing diagnosis is a crucial step to develop an action plan for the care process of the patient. Therefore, the nurse has this very important role with SAE (Systematization of Nursing Care), as it deals with one of its several attributions.
\end{abstract}

Keywords: Mucopolysaccharidosis VI; Maroteaux-Lamy Syndrome; MPS-VI; Nurse.

\title{
Resumen
}

Objetivo: Destacar las necesidades de los pacientes con Síndrome de Maroteaux-Lamy o mucopolisacaridosis VI, y delante de eso proponer posibles diagnósticos de enfermería. Metodología: La investigación es de carácter básico, descriptivo, revisión bibliográfica. En este sentido, se recolectaron datos de artículos en Scielo, BVS y LILACS, entre los años 2002 a 2015, donde durante la recolección de datos se encontraron 1242 artículos, y durante el proceso de selección, luego de todos los filtros, quedaron 7 artículos en total. Por tanto, de estos 7 artículos se recogió la información necesaria para elaborar un marco de posibles diagnósticos de enfermería. Resultado: Los pacientes con MPS VI (mucopolisacaridosis VI) tienen una mayor prevalencia de afectación osteoarticular, según los datos recogidos y analizados. Por lo tanto, los posibles diagnósticos de enfermería pueden ser movilidad física alterada, alteración para caminar, alteración de la transferibilidad, alteración para levantarse, alteración para sentarse. Conclusión: Con base en las manifestaciones clínicas encontradas en la revisión de la literatura, los posibles diagnósticos de enfermería apuntan a maximizar la efectividad del cuidado de enfermería, ya que el diagnóstico de enfermería es un paso crucial para desarrollar un plan de acción para el proceso de cuidado del paciente. Por lo tanto, la enfermera tiene este papel muy importante con SAE (Sistematización de la atención de enfermería), ya que se ocupa de una de sus varias atribuciones.

Palabras clave: Mucopolisacidosis VI; Síndrome de Maroteaux-Lamy; MPS-VI; Enfermero.

\section{Introdução}

A síndrome de Maroteaux-Lamy, também conhecida como mucopolissacaridose tipo VI, é uma síndrome que faz parte de um grupo de doenças metabólicas raríssimas, e afeta um pequeno número de indivíduos, quando comparado com a população geral, ou seja, a incidência mundial é estimada em 1:248.000 a 1:300.000 nascidos vivos entre as mais diferentes populações. Logo são poucos os casos registrados e conhecidos no mundo. Além disso, o Brasil é o país atualmente que contém o maior número de casos registrados no mundo, aonde sua maioria se encontra no nordeste do país (Couto et al., 2016).

No Brasil, nesses últimos vinte anos foram realizados estudos sobre a epidemiologia das MPS dentro do território nacional, durante esse estudo foram diagnosticados cerca de 502 casos de MPS, onde na sua maioria era MPS - II (Síndrome de Hunter), porém a MPS - VI (Síndrome de Maroteaux-Lamy), que é a doença abortada neste estudo, foi a segunda com o maior número de casos no país, onde obteve uma prevalência de cerca de 1,02 em relação as crianças nascidas vivas nesse espaço de tempo que foi realizado o estudo (Federhen et al., 2016).

A MPS VI é pouco conhecida, mas sabe-se que ela foi descrita pela primeira vez em 1963, apresentando algumas semelhanças com a do tipo I e funções cognitivas preservadas, porém há um aumento na excreção urinária do GAG dermatansulfato. Além disso, é uma doença autossômica recessiva, na qual sua causa tem relação com a atividade deficiente da hidrolase lisossômica $\mathrm{N}$-acetilgalactosamina 4-sulfatase, ou arilsulfatase B, onde é codificada pelo gene ARSB, que se localiza no cromossomo 5q13-14 (Cardoso-Santos et al., 2008). 
Bem como, a mucopolissacaridose tipo VI tem como principal característica a deficiência da enzima Nacetilgalactosamina 4-sulfatase, que degrada uma glicosaminoglicano específico, no caso o sulfato de dermatan, como sabemos ocorre um acúmulo intralisossomal dessa molécula, onde consequentemente irá gerar danos celulares que como resultado, teremos diversas alterações multissistêmicas, com caráter crônico e progressivo, além disso, as modificações acontecido por decorrência da síndrome são irreversíveis (Santos et al., 2011).

O tratamento mais indicado para paciente como MPS, é reposição enzimática (TRE), no caso a sua administração é feita periodicamente por via endovenosa, ou seja, é feita a reposição da enzima deficiente. Nesse sentido, a primeira TRE para tratamento de mucopolissacaridose foi para a MPS-I, mas logo em seguida foram aprovadas para MPS-II e MPS-VI (Giugliani et al., 2010).

Nesse sentido, estabelecer um vínculo entre o paciente e o profissional é essencial, assim alavancando a qualidade do atendimento. Já que é crucial o bom relacionamento entre o enfermeiro e o público, ou seja, o profissional deve passar segurança e conforto para o paciente, assim conhecendo bem os clientes, a melhor forma disso acontecer é simplesmente ouvindo atentamente e prestando bem atenção no comportamento de cada indivíduo. Logo, um bom acolhimento faz toda a diferença, já que a falta dele podem provocar a desistência para adesão ao tratamento ou o seu abandono no meio, então é fundamental que o pacienta seja escutado da forma adequado para um cuidado satisfatório. (Camillo \& Maiorino, 2012)

Nesse caso, o processo comunicativo da equipe de enfermagem precisa ser ótimo, ou seja, uma equipe com comunicação não efetiva, nunca conseguirá alcançar um padrão de excelência no processo de cuidar, no momento que a ação da fala é considerada falha, o que mais é prejudicado é o cliente. Logo, enfermeiro e técnicos de enfermagem precisão se comunicar uns com os outros, visto que, no momento de uma intercorrência é necessário que toda a equipe tenha o conhecimento, para assim prevenir futuros complicações no cuidado ao paciente. (Broca \& Ferreira, 2012)

Além disso, é crucial o acompanhamento de uma equipe de enfermagem, para potencializar o cuidado do paciente, nesse caso, o enfermeiro poderá realizar os diagnósticos de enfermagem, já que é umas das etapas do processo de enfermagem, onde é uma ferramenta que possibilita um plano de ação. Nesse sentido, os diagnósticos são feitos a partir dos dados coletados durante a anamnese e exame físico do paciente, assim permite detectar os principais problemas, logo com base nas informações colhidas, esta etapa é primordial para descrever a o cuidado assistencial a prática clínica. (Foschiera \& Viera, 2004)

Ademais o diagnóstico de enfermagem é uma etapa complexa, em razão disso, no caso de um paciente com mucopolissacaridose tipo VI é necessária uma abordagem holística, ou seja, o diagnóstico de enfermagem proporciona uma avalição do comportamento do corpo, onde possibilita interpretar as respostas humanas de uma forma mais eficaz, já que é necessário que o profissional tenha um pensamento crítico e científico, objetividade e iniciativa para tomada de decisão. (França et al., 2007)

Portanto, é preciso ser realizada uma análise profunda de todas as necessidades básicas do paciente que foram afetadas, além disso, há uma série de competências que podem intervir no diagnóstico do enfermeiro, logo, o profissional deve ter a reunir e interpretar os dados coletados, intelecto, capacidade de observação, referencial teórico, experiência, entre outras competências. (França et al., 2007)

\section{Metodologia}

O presente estudo trata-se de uma revisão bibliográfica, de cunho descritivo, do tipo revisão integrativa, onde será realizado um levantamento de dados por etapas com são identificação, triagem, elegibilidade e inclusão, com intuito de usar as informações colhidas para a elaboração de um quadro com os possíveis diagnósticos de enfermagem a partir de artigos 
científicos já desenvolvidos e tem como título Cuidados de enfermagem ao paciente com mucopolissacaridose VI: uma revisão integrativa da literatura.

Para que fosse determinada a questão do estudo foi usada uma pergunta norteadora: $\mathrm{O}$ se tem atualmente produzido na literatura sobre a Síndrome de Maroteaux-Lamy e o que a comunidade acadêmica tem de conhecimento da doença? Também foi determinado os critérios de exclusão: texto incompleto, texto em inglês, texto em outros idiomas, texto com conteúdo que não tivesse alguma relevância com o tema a ser abordado. Além disso, os critérios de inclusão também foram estabelecidos: texto completo, gratuito, texto em português, texto com conteúdo de relevância com o tema a ser abordado.

No início de dezembro de 2020 foram feitas as buscas dos artigos usando as seguintes bases eletrônicas de dados: SciELO, LILACS e BVS, e foram utilizados os descritores: MPS-VI, mucopolissacaridose VI e Síndrome de MaroteauxLamy, Doenças raras. Onde foram encontrados cerca de 1242 artigos, na qual durante o processo de seleção restou apenas 7 artigos para serem revisados. Logo, desses selecionados que serviram de suporte de metodológico, autores como Ana Carolina de Paula (Paula et al. 2006), Celso Montenegro Turtelli (Turtelli, 2002), entre outros grandes nomes da comunidade acadêmica.

Foram incluídos artigos com pesquisas publicadas nos últimos o vinte (20) anos, artigos originais, em língua portuguesa. Serão excluídos os artigos que estiverem incompletos, artigos que abordem sobre outros tipos de síndrome, artigos em outros idiomas, artigos repetidos e que tenham data de publicação anterior há vinte (20) anos. A tabela dos possíveis diagnósticos de enfermagem será elaborada de acordo com as manifestações clínicas esplanadas em cada artigo científico já desenvolvimento.

Em seguida, após a coleta e análise das evidências foi elaborado um quadro com os possíveis diagnóstico de enfermagem, visto que, o diagnóstico de enfermagem é uma competência exclusiva do enfermeiro, já que é umas das etapas do SAE e que é crucial para todas elaboração das intervenções de enfermagem. Vale ressaltar que no estudo está sendo proposto algumas possíveis diagnósticos para os pacientes com MPS VI, já pode mudar de caso para caso, pois a conduta pode mudar conforme o quadro clínico do cliente. Assim, havendo a necessidade de uma adaptação de um paciente para o outro.

Logo abaixo encontra-se um Fluxograma onde ilustra todo os passos do processo de seleção dos artigos escolhidos para o estudo, onde primeiramente foi quantificado os resultados de achados em cada base de dados e também filtrados conforme dos critérios estabelecidos para o estudo, após foram feitas mais uma série de análises minuciosas para se chegar a um resultado que fosse até para revisão integrativa de literatura, confira a seguir. 
Figura 1. Fluxograma de busca nas bases de dados para revisão integrativa sobre a Síndrome de Maroteaux-Lamy.

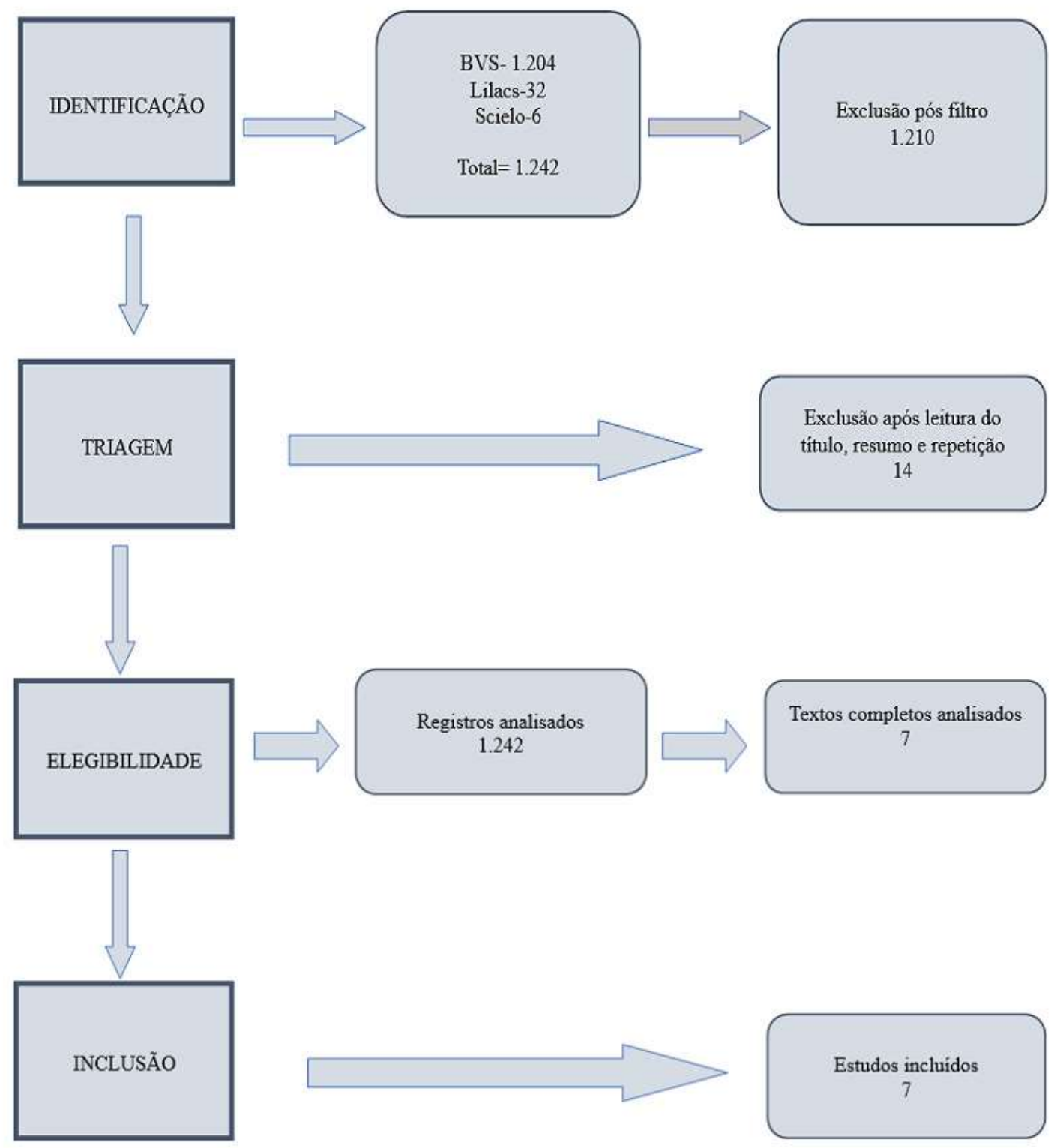

Fonte: Autores.

\section{Resultados}

A mucopolissacaridose VI é uma doença genética metabólica, onde apresenta uma grande quantidade de sinais e sintomas, isso pelo fato de ser uma síndrome, e logo a seguir está uma quadro com algumas das manifestações clínicas encontradas em cada artigo, vale ressaltar que algumas manifestações podem ser citadas por dois ou mais artigos diferentes, nesse caso, o artigo que já houver citado o sinal clínico, não irá ser citado novamente no quadro, mas serão mencionados apenas os que ainda não estão no Quadro a seguir. 
Quadro 1 - Manifestações Clínicas encontras em pacientes portadores de Mucopolissacaridose tipo VI (síndrome de MaroteauxLamy) nos estudos publicados entre os anos de 2002 a 2015.

\begin{tabular}{|c|c|c|c|}
\hline Autor e Ano & $\begin{array}{c}\text { Metodologia / Nível de } \\
\text { Evidência }\end{array}$ & Título & Manifestações Clínicas \\
\hline $\begin{array}{l}\text { Cardoso-Santos, A. } \\
\text { et al. } \\
2008\end{array}$ & Evidência 3B & $\begin{array}{l}\text { Mucopolissacaridose Tipo VI (síndrome de } \\
\text { Maroteaux-Lamy): avaliação da } \\
\text { mobilidade articular e das forças de garra e } \\
\text { de pinça }\end{array}$ & $\begin{array}{l}\text { - } \text { Mãos em garra; } \\
\text { - } \quad \text { Síndrome do túnel do carpo; } \\
\text { - } \text { Incapacidade de realizar o } \\
\text { movimento de pinça; } \\
\text { - } \\
\text { Deficiência das necessidades } \\
\text { simples; } \\
\text { - } \quad \text { Fibrose da cápsula articular; } \\
\text { - } \quad \text { Movimentos limitados. }\end{array}$ \\
\hline $\begin{array}{l}\text { Turtelli, C. M. } \\
2002\end{array}$ & $\begin{array}{l}\text { Relato de Caso } \\
\text { Evidência } 4\end{array}$ & $\begin{array}{l}\text { Manifestações radiológicas da } \\
\text { mucopolissacaridose Tipo VI }\end{array}$ & $\begin{array}{ll}\text { - } & \text { Deformidade de membros e pele } \\
\text { espessa; } \\
\text { - } & \text { Face gargólica; } \\
\text { - } & \text { Crânio com abaulamento frontal; } \\
\text { - } & \text { Cifose toracolombar; } \\
\text { - } & \text { Corpos vertebrais hipoplásicos; } \\
\text { - } & \text { Densificação e irregularidade das } \\
\text { - } & \text { Hífises; } \\
\text { - } & \text { Estreitamento do canal vertebral na dos ilíacos; } \\
\text { - } & \text { Alteransição craniovertebral; } \\
\text { - } & \text { lesions"; } \\
\text { Áreas hiperintensas em } \mathrm{T} 2 .\end{array}$ \\
\hline $\begin{array}{l}\text { Paula, A. C. D. et al. } \\
2006\end{array}$ & $\begin{array}{l}\text { Estudo de Caso } \\
\text { Evidência 3B }\end{array}$ & $\begin{array}{c}\text { Achados radiológicos em pacientes com } \\
\text { mucopolissacaridose Tipo VI } \\
\text { (Síndrome de Maroteaux-Lamy) }\end{array}$ & 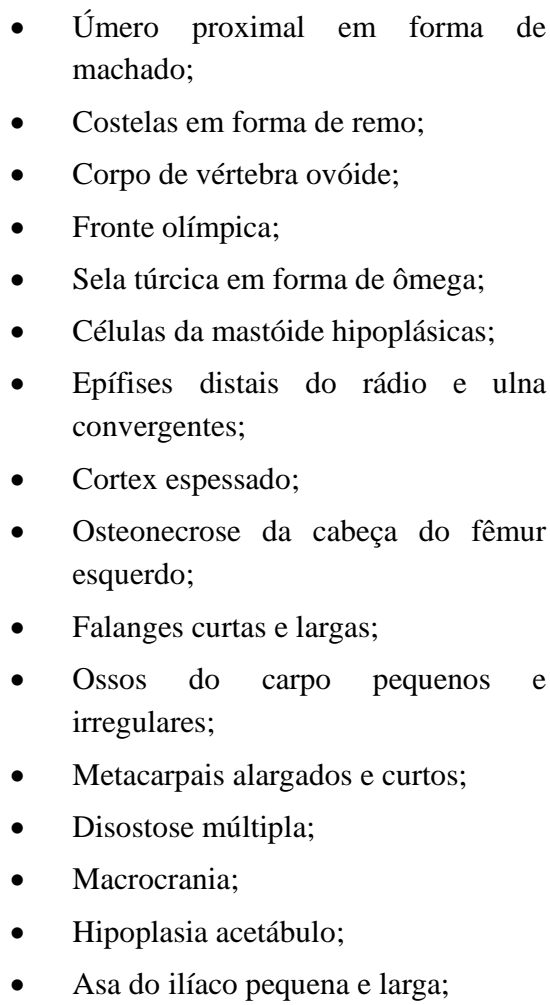 \\
\hline
\end{tabular}


Research, Society and Development, v. 11, n. 1, e2211123435, 2022

(CC BY 4.0) | ISSN 2525-3409 | DOI: http://dx.doi.org/10.33448/rsd-v11i1.23435

\begin{tabular}{|c|c|c|c|}
\hline & & & - Ossos tubulares curtos; \\
\hline $\begin{array}{l}\text { Canêdo, M. G. R. } \\
\text { D. R. et al. } \\
2006\end{array}$ & $\begin{array}{l}\text { Estudo de Caso } \\
\text { Evidência } 4\end{array}$ & $\begin{array}{c}\text { Pseudoglaucoma em } \\
\text { mucopolissacaridose Tipo VI: } \\
\text { relato de caso }\end{array}$ & 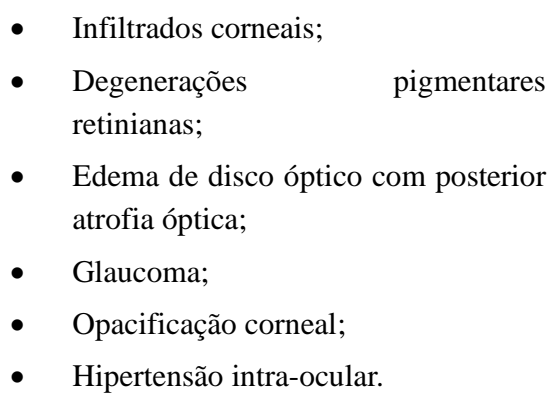 \\
\hline $\begin{array}{l}\text { Mizuno, C. A. et al. } \\
2010\end{array}$ & $\begin{array}{c}\text { Revisão Integrativa de } \\
\text { Literatura }\end{array}$ & $\begin{array}{l}\text { Aspectos clínicos da } \\
\text { mucopolissacaridose Tipo VI }\end{array}$ & 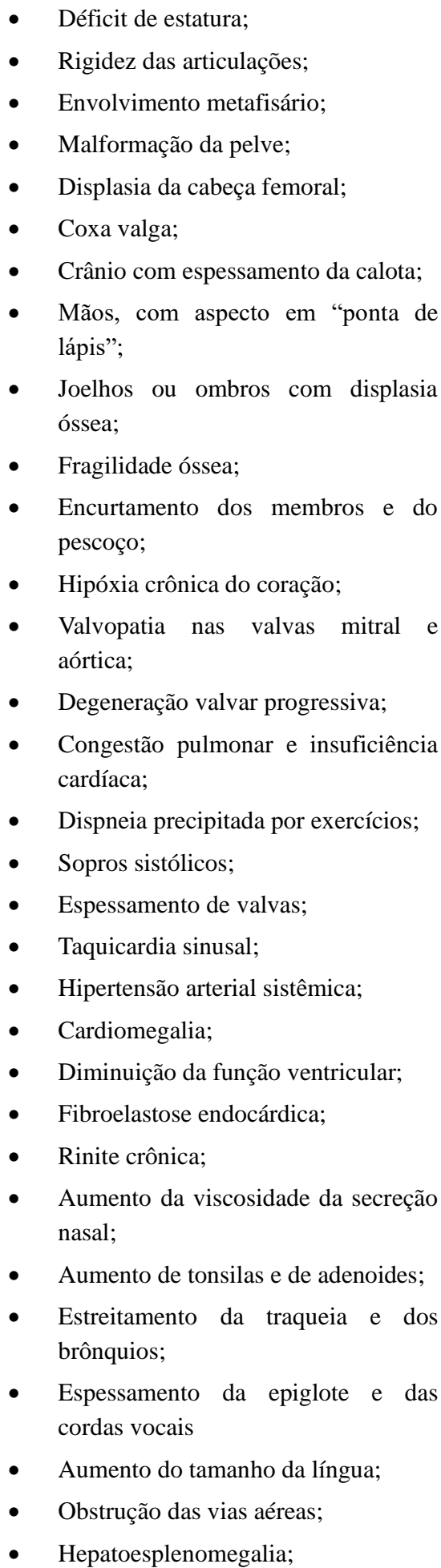 \\
\hline
\end{tabular}




\begin{tabular}{|c|c|c|c|}
\hline & & & $\begin{array}{ll}\text { - } & \text { Pequeno desvio na coluna torácica; } \\
\text { - } & \text { Aumento na lordose lombar; } \\
\text { - } & \text { Apneia Obstrutiva do Sono; } \\
\text { - } & \text { Sonolência diurna; } \\
\text { - } & \text { Hipertensão pulmonar; } \\
\text { - } & \text { Perda auditiva neurossensorial; } \\
\text { - } & \text { Catarata; } \\
\text { - } & \text { Degeneração vítrea; } \\
\text { - } & \text { Papiledema; } \\
\text { - } & \text { Atrofia ótica; } \\
\text { - } & \text { Ptose; } \\
\text { - } & \text { Hidrocefalia; } \\
\text { - } & \text { Espessamento da dura-máter; } \\
\text { - } & \text { Disfunção da aracnoide; } \\
\text { - } & \text { Atrofia cortical; } \\
\text { - } & \text { Dores de cabeça diurnas e vômitos; } \\
\text { - } & \text { Deterioração visual; } \\
\text { - } & \text { Fraqueza; } \\
\text { - } & \text { Instabilidade atlantoaxial ; } \\
\text { - } & \text { Aumento do perímetro cefálico; } \\
\text { - } & \text { Hérnias umbilicais e inguinais; }\end{array}$ \\
\hline $\begin{array}{l}\text { Gomes, B. S. D. et } \\
\text { al } \\
2011\end{array}$ & $\begin{array}{l}\text { Estudo de Caso } \\
\text { Evidência 3B }\end{array}$ & $\begin{array}{l}\text { Manifestações cardíacas em pacientes } \\
\text { com mucopolissacaridose vi }\end{array}$ & $\begin{array}{l}\text { - } \quad \text { Desvio do eixo cardíaco para direita; } \\
\text { - } \quad \text { Deformidade torácica; } \\
\text { - } \text { distúrbios de condução; } \\
\text { - Lesões cardíacas; } \\
\text { - Lesão valvular; } \\
\text { - } \\
\text { Estenose e regurgitação; } \\
\text { anterior da válvula mitral. }\end{array}$ \\
\hline $\begin{array}{l}\text { Medeiros, J. N. S. et } \\
\text { al. } \\
2015\end{array}$ & $\begin{array}{l}\text { Relato de Caso } \\
\text { Evidência 3B }\end{array}$ & $\begin{array}{l}\text { Avaliação da força de preensão e } \\
\text { amplitude de movimentos dos } \\
\text { membros superiores em pacientes com } \\
\text { mucopolissacaridose VI }\end{array}$ & $\begin{array}{l}\text { - } \quad \text { Dor em membros superiores; } \\
\text { - Dor progressiva. }\end{array}$ \\
\hline
\end{tabular}

Fonte: Autores.

A partir dos dados coletados do quadro anterior, foi elaborado um segundo quadro com os possíveis diagnósticos de enfermagem, onde todos estão conforme o NANDA, vale ressaltar que os possíveis diagnósticos, ou seja, são algumas sugestões, pois o cuidado do paciente é individualizando de acordo com sua situação clínica. Logo, a seguir o Quadro 2. 
Quadro 2 - Proposta de diagnósticos de enfermagem voltados à Síndrome de Maroteaux-Lamy segundo as manifestações clínicas encontradas na literatura entre os anos de 2002 e 2015.

\begin{tabular}{|c|c|c|}
\hline DOMINIO-CLASSE & DIAGNÓSTICO & $\begin{array}{c}\text { MANISFESTAÇÕES CLÍNICAS } \\
\text { EVIDÊNCIA }\end{array}$ \\
\hline Nutrição - Ingestão & $\begin{array}{l}\text { Risco de deglutição prejudicada relacionado a } \\
\text { anormalidade de via aérea superior. }\end{array}$ & $\begin{array}{l}\text { - } \quad \text { Espessamento da epiglote e das cordas vocais; } \\
\text { - } \quad \text { Aumento do tamanho da língua. }\end{array}$ \\
\hline Nutrição - Hidratação & Volume de líquidos deficiente & $\begin{array}{l}\text { - } \quad \text { Taquicardia; } \\
\text { - } \quad \text { Fraqueza; } \\
\text { - } \quad \text { Perda de peso. }\end{array}$ \\
\hline Nutrição - Hidratação & Volume de líquidos excessivo relacionado & $\begin{array}{ll}\text { - } & \text { Hipertensão arterial sistêmica; } \\
\text { - } & \text { Dispneia; } \\
\text { - } & \text { Congestão pulmonar; }\end{array}$ \\
\hline $\begin{array}{l}\text { Eliminação e Troca - Função } \\
\text { respiratória }\end{array}$ & Troca de gases prejudicada & $\begin{array}{l}\text { - } \quad \text { Dispneia; } \\
\text { - } \quad \text { Sonolência diurna; } \\
\text { - } \quad \text { Hipóxia. }\end{array}$ \\
\hline $\begin{array}{l}\text { Atividade/ Repouso } \quad- \\
\text { Atividade/ Exercícios }\end{array}$ & $\begin{array}{ll}\text { - } & \text { Capacidade de transferência prejudicada } \\
\text { - } & \text { Deambulação prejudicada } \\
\text { - } & \text { Leavantar-se prejudicado } \\
\text { - } & \text { Mobilidade física prejudica } \\
\text { - } & \text { Sentar-se prejudicado }\end{array}$ & $\begin{array}{l}\text { - } \quad \text { Atrofia ótica e perda da visão; } \\
\text { - } \quad \text { Glaucoma; } \\
\text { - } \quad \text { Catarata; } \\
\text { - } \quad \text { Fraqueza das extremidades inferiores, paraplegia } \\
\text { espástica ou quadriplegia; } \\
\text { - } \quad \text { Incapacidade de realizar o movimento de pinça; } \\
\text { - } \quad \text { Déficit de estatura; } \\
\text { - } \text { Baixa estatura, disostose múltipla, e limitação } \\
\text { - } \quad \text { articular; } \\
\text { - } \quad \text { Rigidezimentos dimitados; } \\
\text { - } \text { Apresenta marcha nas pontas dos pés. }\end{array}$ \\
\hline $\begin{array}{l}\text { Atividade/ Repouso - } \\
\text { Respostas Cardiovasculares/ } \\
\text { Pulmonares }\end{array}$ & $\begin{array}{l}\text { - } \quad \text { Débito cardíaco diminuído } \\
\text { - } \quad \text { Risco de função cardiovascular prejudicada } \\
\text { relacionado a hipertensão arterial. }\end{array}$ & $\begin{array}{ll}\text { - } & \text { Dispneia; } \\
\text { - } & \text { Fadiga; } \\
\text { - } & \text { Taquicardia; } \\
\text { - } & \text { Hipertensão Arterial Sistêmica; }\end{array}$ \\
\hline $\begin{array}{l}\text { Atividade/ Repouso - } \\
\text { Respostas Cardiovasculares/ } \\
\text { Pulmonares }\end{array}$ & Padrão respiratória ineficaz & $\begin{array}{l}\text { - } \quad \text { Dispneia; } \\
\text { - } \quad \text { Fadiga; } \\
\text { - } \quad \text { Jão em garra; } \\
\text { - } \quad \text { Fragilidade óssea; } \\
\text { - } \quad \text { Encurtamento dos membros e do pescoço; } \\
\text { - } \quad \text { Úmero proximal em forma de machado; } \\
\text { - } \quad \text { Costelas em forma de remo; } \\
\text { - } \quad \text { Corpo de vértebra ovóide; } \\
\text { - } \quad \text { Fronte olímpica com fechamento precoce das } \\
\text { - } \quad \text { Suturas; } \\
\text { - } \quad \text { Exífises dírcica em forma de ômega; } \\
\end{array}$ \\
\hline
\end{tabular}




\begin{tabular}{|c|c|c|c|}
\hline & & & $\begin{array}{l}\text { - Osteonecrose da cabeça do fêmur esquerdo; } \\
\text { - } \quad \text { Falanges curtas e largas nas diáfises extremidades } \\
\text { proximais; } \\
\text { - Ossos do carpo pequenos e irregulares; } \\
\text { - } \quad \text { Macrocrania. }\end{array}$ \\
\hline $\begin{array}{ll}\text { Atividade/ Repouso } \\
\text { Autocuidado }\end{array}$ & & $\begin{array}{l}\text { - } \quad \text { Déficit no autocuidado para banho. } \\
\text { - } \quad \text { Déficit no autocuidado para higiene íntima. } \\
\text { - } \quad \text { Déficit no autocuidado para vestir-se }\end{array}$ & $\begin{array}{ll}\text { - } & \text { Dor progressiva; } \\
\text { - } & \text { Fraqueza das extremidades inferiores, paraplegia } \\
\text { - } & \text { Dor em membrica ou quadriplegia. } \\
\text { - } & \text { Perda de massa muscular; } \\
\text { - } & \text { Fadiga; } \\
\text { - } & \text { Rigidez nas articulações }\end{array}$ \\
\hline $\begin{array}{l}\text { Crescimento/ } \\
\text { Desenvolvimento } \\
\text { Desenvolvimento }\end{array}$ & - & $\begin{array}{l}\text { Risco de desenvolvimento atrasado relacionado } \\
\text { por visão prejudicada e distúrbios genéticos. }\end{array}$ & $\begin{array}{ll}\text { - } & \text { Infiltrados corneais; } \\
\text { - } & \text { Opacificação progressiva da córnea; } \\
\text { - } & \text { Degenerações pigmentares retinianas; } \\
\text { - } & \text { Catarata; } \\
\text { - } & \text { Degeneração vítrea; } \\
\text { - } & \text { Hipertensão ocular; } \\
\text { - } & \text { Glaucoma. }\end{array}$ \\
\hline
\end{tabular}

Fonte: Autores.

\section{Discussão}

No estudo realizado por Santos et. al (2008) percebeu-se que há um grande empenho de flexão de ombro, sem ligação com a idade, e da extensão de joelho e flexão de cotovelo, estas por sua vez são relacionadas de forma negativa com a idade. A força de garra apresentou-se envolvida em todos os pacientes, e a força da pinça evidenciou uma relação positiva com idade.

As mudanças clínico-radiológicas começam entre dois e quatro anos de idade, e as mais relevantes são: atraso no crescimento, cifose toracolombar, hepatoesplenomegalia e rigidez das articulações. As modificações na RM são variáveis. Com o progresso da doença, pode acontecer mudança de sinal na Substância branca periventricular. Esse fato acontece devido aos resíduos de mucopolissacarídeos nos espações perivasculares (Turtelli, 2002)

Segundo Borges et al (2003) os indivíduos com MPS podem manifestar dolicocefalia, fronte ampla e deformações na coluna mesmo sendo considerados normais ao nascer. Maior parte dos pacientes desenvolve os primeiros sinais da doença ainda na infância (hepatoesplenomegalia, face infiltrada e atraso de crescimento) se apresentando entre 1 e 3 anos de idade.

Como é uma doença crônica, os apanhados clínicos pioram-se com os anos. Os pacientes começam a desenvolver mudanças em vários tecidos e órgãos, podendo ser desde mudanças osteoarticulares como também alterações cardiorrespiratórias, uma vez que esses distúrbios são gradativos e podem levar o paciente ao óbito.

Conforme o estudo de Gomes et. al (2011) nem sempre com realização do exame físico é possível analisar o grau de comprometimento cardíaco, principalmente na sua fase inicial. Portanto, é crucial o eletrocardiograma para avaliação todo paciente.

Há também acometimento do endocárdio, por sua vez, afeta as válvulas cardíacas, visto que acontece a infiltração de GAG's, que a partir do momento que ocorre sua acumulação. Além do que, atinge também o sistema de condução e no miocárdio, dando origem as miocardiopatias restritivas e também distúrbios de condução. (Gomes et al., 2011)

Segundo Medeiros et. al (2015) no seu estudo notou que a função articular dos indivíduos com MPS-VI é classificada com anômala devido ao espessamento e fibrose da capsula articular, originando restrições de grandes articulações, assim 
também nas menores articulações, por exemplo a dos dedos e acaba acarretando na mão em garra. Além disso, é frequente que ocorra a síndrome do túnel do carpo em paciente da síndrome de Matoteaux-Lamy.

A dor progressiva existente nas articulações e inexistente de mobilidade articular, é um fator importantíssimo para morbidade nesses pacientes. O estudo percebeu uma forte dor nas articulações de ombros, cotovelos e punhos, onde é uma das articulações mais apontadas pelos pacientes, como mais doloridas MT- CFs, IFPs e IFDs. (Medeiros et al., 2015)

Visto que quanto maior a idade do paciente, menor é a mobilidade. Dessa forma, a análise da mobilidade articular do joelho, quadril e cotovelo, podem ser vistos como um ótimo marcador do desenvolvimento da MPS VI.

Pacientes com deformidade na pelve, displasia da cabeça femoral e coxa valga tem uma propensão de progredir doenças evoluídas e debilitadoras do quadril.

\section{Considerações Finais}

Portanto, se um paciente de Mucopolissacaridose VI que apresenta sinais e sintomas como: rigidez articular, alteração na marcha, falta de ar ao fazer esforço, alterações ósseas, baixa estatura e dor realizar movimentos. Logo, a partir dessas informações coletadas. Logo, o possível diagnóstico de enfermagem é "Mobilidade física prejudicada relacionada a Síndrome de Maroteaux-Lamy, evidenciado por dispneia ao esforço, alteração na marcha, alteração na integridade de estrutura óssea, atraso de desenvolvimento, dor e rigidez articular. "Como foi relato nessa revisão integrativa o paciente diagnosticado com a Síndrome de Maroteaux-Lamy tem diversas complicações ao longo da vida, mas com um profissional de enfermagem capacitado é possível ofertar um cuidado que minimize suas dificuldades do dia a dia, como foi discorrido nesse estudo, as pessoas com MPS VI têm uma certeza uma predisposição até uma mobilidade física prejudicada.

Para finalizar esse estudo, vale ressaltar que a doença ela se apresenta das mais diversas formas em um paciente, ou seja, um paciente $\mathrm{X}$ pode apresentar baixa estatura, rigidez articular, marcha alterada e mão garra, mas no paciente $\mathrm{Y}$ a doença se manifesta com cardiomegalia, glaucoma, hipertensão, congestão pulmonar, face gargólica e rinite crônica. Nesse sentido, os diagnósticos de enfermagem podem ser adaptados conforme os sinais e sintomas do paciente, logo, os cuidados de enfermagem não são os mesmo para os pacientes. Além disso, há outras manifestações clinicas como: Dispneia, hidrocefalia, sopro sistólico, taquicardia, úmero em forma de machado, costelas em forma de remo, hipertensão ocular, dor progressiva, fadiga, insuficiência cardíaca, catarata, atrofia cortical, perda de peso, deterioração visual, síndrome do túnel do carpo, coxa valga, incapacidade de realizar o movimento de pinça, movimentação limitada e entre outros diversos citados no estudo.

\section{Referências}

Foschiera, F., \& Viera, C. S. (2004). O diagnóstico de enfermagem no contexto das ações de enfermagem: percepção dos enfermeiros docentes e assistenciais. Revista Eletrônica de Enfermagem, 06 (02), 189-198. http://www.revistas.ufg.br/index.php/fen

França, F. C. D. V., Kawaguchi, I. A. L., Silva, E. P. D., Abrão, G. A., Uemura, H., Alfonso, L. M., \& Carvalho, E. O. D. (2007). Implementação do diagnóstico de enfermagem na unidade de terapia intensiva e os dificultadores para enfermagem - relato de experiência. Revista Eletrônica de Enfermagem, 09 (02), 537-456. http://www.fen.ufg.br/revista/v9/n2/v9n2a20.htm

Fontes, C. M. B., \& Cruz, D. D. A. L. M. D. (2007). Diagnósticos de enfermagem documentados para pacientes de clínica médica. Revista da Escola de Enfermagem da USP, 41 (3), 395-402. https://doi.org/10.1590/S0080-62342007000300008

Turtelli, C.M. (2002). Manisfestações radiológicas da mucopolissacaridose tipo VI. Radiologia Brasileira, 35 (5). https://doi.org/10.1590/S010039842002000500013

Paula, A. C. D., Bertola, D. R., Albano, L. M. J., Santos, A. C. G. M. D., Donato, F. J., Oliveira, L. A. N. D., \& Kim, C.A. (2006). Achados radiológicos em pacientes com mucopolissacaridose tipo VI (Síndrome de Maroteaux-Lamy). Revista Imagem, 28 (1), 7-12. https://web.archive.org/web/20090806172929/http://www.spr.org.br:80/files/public/magazine/public_87/07.pdf

Cardoso-Santos, A., Azevedo, A. C. M. M., Fagondes, S., Burin, M. G., Giugliani, R., \& Schwartz, I. V. D. (2008). Mucopolissacaridose tipo VI (síndrome de Maroteaux-Lamy): avaliação da mobilidade articular e das forças de garra e de pinça. Jornal de Pediatria, 84 (2), 130-135. https://doi.org/10.1590/S002175572008000200007 
Santos, A. S. D., Santos Neto, F. P. D., Silva, D. A., \& Souza, D. O. (2011). Mucopolissacaridose tipo VI (Síndrome de Maroteaux Lamy): relato de caso. Revista de Ciências Médicas e Biológicas,10 (2), 194-197. https://portalseer.ufba.br/index.php/cmbio/article/view/5250/4175

Giugliani, R., ..., \& Martins, A. M. (2010). Terapia de reposição enzimática para as mucopolissacaridoses I, II e VI: recomendações de um grupo de especialistas brasileiros. Revista da Associação Médica, $56 \quad$ (3), 271-277.http://www.scielo.br/scielo.php?script=sci_arttext\&pid=S0104$42302010000300009 \& \operatorname{lng}=\mathrm{en} \& \mathrm{nrm}=\mathrm{iso}$

Federhen, A., Giugliani, R., \& Martins, U. (2017). Mucopolissacaridoses: um estudo abrangente sobre a epidemiologia da doença no Brasil. Tese de doutorado, Programa de Pós-graduação em saúde da criança e do adolescente, Faculdade de medicina, Universidade Federal do Rio Grande do Sul. Porto Alegre, Brasil. https://lume.ufrgs.br/handle/10183/211283

Couto, R. R. S. D., Bochernitsan, A. N., Bender, F., Facchin, A. C. B., Trapp, F., Dick, J., Leistner-Segal, S. (2016). Atualização das frequências de mutações no gene ARSB em pacientes com Mucopolissacaridose tipo VI após 10 anos de análises. Clinical and biomedical research, 36 (Supl.). https://www.lume.ufrgs.br/bitstream/handle/10183/164448/001007041.pdf?sequence=1

Camillo, S. D. O., \& Maiorino, F. T. (2012). A importância da escuta no cuidado de enfermagem. Revista Gogitare Enfermagem. 17 (3), 549-555. https://revistas.ufpr.br/cogitare/article/view/27826/19049

Broca, P. V., Ferreira, M. D. (2012). Equipe de enfermagem e comunicação: contribuições para o cuidado de enfermagem. Revista Brasileira de Enfermagem, 65 (1). https://doi.org/10.1590/S0034-71672012000100014

Canêdo, M. G. R. D. R., Almeida, L. N. F. D., Silva, R. G. D., Almeida, R. N. F. D., \& Alessandri, E. F. (2006). Pseudoglaucoma em mucopolissacaridose tipo VI: relato de caso. Arquivos Brasileiros de Oftalmologia, 69 (6). https://doi.org/10.1590/S0004-27492006000600026

Mizuno, C. A., Figueiredo, J. B., Teza, I. T. V., Taira, L. G. N., Paixão, D. L., \& Mizuno, J. C. (2010). Aspectos clínicos da mucopolissacaridose tipo VI. Revista da Sociedade Brasileira de Clínica Medica, 8 (4), 356-361. http://files.bvs.br/upload/S/1679-1010/2010/v8n4/a013.pdf

Gomes, B. S. D., Nóbrega, C. E. B. D., Medeiros, P. F. V. D., Medeiros, I. A. M. C. D. Pordeus, A. C. B. (2011). Manifestações cardíacas em paciente com $\begin{array}{lllll}\text { mucopolissacaridose } & \text { VI. } & \text { Revista } & \text { (1), } & \text { Ecocardiografia, }\end{array}$ http://departamentos.cardiol.br/dic/publicacoes/revistadic/revista/2011/portugues/Revista01/12-manifestacoes.pdf

Medeiros, J. N. S. D., Silva, B. B. R. D., Queiroz, D. D. R., Cattuzzo, M. T., Soares, K. M. S. (2015). Avaliação da força de preensão e amplitude de movimentos dos membros superiores em pacientes com mucopolissacaridose V. Revista Acta Fisiátrica, 22 (2), 60-64. https://www.revistas.usp.br/actafisiatrica/article/view/114500/112328

Borges, M. F., Tavares, F. S., Silva, P. C. L., Oliveira, Z. A. R., Ballarin, M. A.S., Gomes, R. A., \& Ferreira, B. P. (2003). Mucopolissacaridose tipo VI (síndrome de Maroteaux-Lamy): avaliação endócrina de três casos. Arquivos Brasileiros de Endocrinologia \& Metabologia, 47 (1), 87-94. http://www.scielo.br/scielo.php?script=sci_arttext\&pid=S0004-27302003000100014\&lng=en\&nrm=iso

Dieter, T., Matte, U., Barbosa, F., Schwartz, I., \& Giugliani, R. (2002). Introdução às mucopolissacaridoses. Universidade Federal do Rio Grande do Sul. http://www.ufrgs.br/redempsbrasil/sobre/introducao_as_mucopossacaridoses.pdf.

Franco, J. F. D. S. (2015). Avaliação clínico-laboratorial dos pacientes com mucopolissacaridose tipos I, II e VI em terapia de reposição enzimática (TRE). Tese de Doutorado. Universidade de São Paulo. https://www.teses.usp.br/teses/disponiveis/5/5141/tde-24032016142203/publico/JoseFranciscodaSilvaFrancoVersaoCorrigida.pdf.

Herdman, T. H., \& Kamitsuru, S. (2015-2017). Diagnósticos de Enfermagem da NANDA: Definição e Classificação. Porto Alegre: Artmed. 